\title{
ESPECIES DE Oithona (CRUSTACEA: COPEPODA) EN EL PACÍFICO COLOMBIANO EN EL SEGUNDO PERIODO LLUVIOSO DE 2001
}

\section{Oithona (CRUSTACEA: COPEPODA) SPECIES IN THE COLOMBIAN PACIFIC IN THE SECOND RAINY PERIOD OF 2001}

\author{
Raúl Hernando López Peralta • Luz Helena Mojica López ${ }^{2,3}$
}

\section{RESUMEN}

La importancia ecológica mundial de los copépodos motivó este primer trabajo en el Pacífico colombiano sobre Oithona, considerado el género más abundante y ubicuo en todos los océanos. Las capturas de los organismos se efectuaron del 23 de junio al 15 de septiembre de 2001 mediante arrastres superficiales con una red cónica, para valorar su abundancia en relación con algunas variables físico-químicas, la hora de obtención y el ciclo lunar. Los taxa identificados fueron O. plumifera (61.8\%), O. rigida (15.0\%), 0. atlantica (8.7\%), 0. nana (5.9\%), O. robusta (2.0\%) y Oithona spp. (6.7\%). El poro de la malla (363 $\mu \mathrm{m})$ explicó el bajo número de copépodos, pues la mayoría mide $<1 \mathrm{~mm}$, excepto 0 . plumifera ( $>1 \mathrm{~mm}$ ), lo cual implicó sus mayores y frecuentes capturas. La variación espacio-temporal general fue concomitante con la heterogeneidad oceanográfica del Pacífico colombiano en las mismas escalas. La abundancia general noche: día (56.3 vs. 43.7\%) sugirió alguna migración vertical circadiana, acentuada en 0 . atlantica, O. rigida y 0 . robusta. Las dos primeras especies y O. plumifera, aumentaron durante luna llena, en parte atribuible a una menor presión depredadora. Los intervalos de temperatura $\left(26.0-29.7^{\circ} \mathrm{C}\right)$, salinidad (27.1-33.6 ups), $\mathrm{O}_{2}(3.8-5.4 \mathrm{mg} / \mathrm{l})$ y clorofila-a $\left(0-3.2 \mathrm{mg} / \mathrm{m}^{3}\right)$ superficiales fueron de condiciones normales, pese al desarrollo del episodio La Niña 1998-2001, detectado en el área el último año, pero sólo subsuperficialmente. El ACP señaló la mayor influencia de la temperatura sobre los copépodos, lo que quizás se relacionó con la disminución general de Oithona en agosto y septiembre.

Palabras clave: Cyclopoida, microcrustáceos, La Niña, variación noche: día.

1 Raúl Hernando López Peralta, Dr. rer. nat. Laboratorio de Hidrobiología, Programa de Biología Aplicada, Facultad de Ciencias Básicas y Aplicadas. Campus Nueva Granada, Universidad Militar Nueva Granada, km 2 vía Cajicá-Zipaquirá, Colombia.

2 Luz Helena Mojica López, Bióloga Marina. Instituto de Investigaciones Marinas y Costeras (INVEMAR), calle 25 No. 2-55 Playa Salguero, Santa Marta, Colombia.

3 Envío de correspondencia: raul.lopez@unimilitar.edu.co. 


\begin{abstract}
The global ecological importance of copepods motivated this first study in the Colombian Pacific on Oithona, considered the most abundant and ubiquitous genus in all oceans. Catches of organisms were from June 23 to September 15, 2001 by surface tows with a conical net, for assessing their abundance in relation to some physicochemical variables, the capture time and the lunar cycle. The taxa identified were O. plumifera (61.8\%) O. rigida (15.0\%), O. atlantica (8.7\%), O. nana (5.9\%), O. robusta (2.0\%), and Oithona spp. (6.7\%). The meshpore $(363 \mu \mathrm{m})$ explained the low number of species and copepods, as the majority measures $<1 \mathrm{~mm}$, except O. plumifera (> $1 \mathrm{~mm}$ ), which implied their highest and frequent catches. The general spatiotemporal variation was concomitant with the Colombian Pacific oceanographic heterogenity in the same scales. The general night: day abundance (56.3 vs. $43.7 \%$ ) suggested a vertical circadian migration accented in $O$. atlantica, $O$. rigi$d a$ and $O$. robusta. The first two species and $O$. plumifera increased during full moon, in part attributable to a minor predation pressure. Temperature $\left(26.0-29.7^{\circ} \mathrm{C}\right.$ ), salinity (27.1-33.6 ups), $\mathrm{O}_{2}(3.8-5.4 \mathrm{mg} / \mathrm{l})$, and chlorophylla $\left(0-3.2 \mathrm{mg} / \mathrm{m}^{3}\right)$ surface ranges were normal, despite the development of La Niña 1998-2001 episode, detected in the area the last year, but only in the subsurface. The ACP indicates the strongest influence of temperature on copepods, perhaps related to the overall decline in Oithona in August and September.
\end{abstract}

Keywords: Cyclopoida, microcrustaceans, La Niña, night: day variation.

\title{
INTRODUCCIÓN
}

Los copépodos son microcrustáceos de abundancia formidable en todos los ecosistemas acuáticos con ca. 2.300 especies planctónicas marinas, muchas del mesozooplancton (200-2000 $\mu \mathrm{m}$ ) (Razouls et al. 2014). La familia Oithonidae Dana, 1853 (orden Cyclopoida) predomina en el zooplancton de los ecosistemas costeros y oceánicos (Paffenhöfer 1993), con una abundancia cíclica en muchos estuarios y bahías tropicales y subtropicales (Ferrari \& Orsi 1984). El género Oithona posee 49 especies que habitan las zonas epi- y mesopelágica de todos los océanos (Razouls et al. 2014). Junto con Corycaeus y Oncaea son los tres géneros de Cyclopoida de tamaño muy pequeño $(<1 \mathrm{~mm}$ ) (Nishida 1985, González et al. 2000).

Desde hace décadas se reconoce a Oithona como uno de los taxa del zooplancton más destacados por diferentes razones: tasas metabólicas bajas períodos reproductivos prolongados, ciclos de vida acoplados con la oferta alimentaria, capacidad de sobrevivir y producir huevos en situaciones de poco alimento (Paffenhöfer 1993), dado su amplio espectro trófico (Lonsdale et al. 2000), función homeostática en el funcionamiento del medio pelágico (Gallienne \& Robins, 2001, Turner 1986, 2004), disminución del flujo vertical de la materia particulada y consecuente incremento de la regeneración de nutrientes en la capa fótica (González \& Smetacek 2004, Almeda et al. 2011), abundancias y biomasas elevadas (Cepeda et al. 2012).

Sin embargo, muchas características básicas de la biología, fisiología y ecología de Oithona son aún poco conocidos, particularmente sus fases ontogénicas tempranas (Gallienne \& Robins 2001, Zamora et al. 2014a, 
2014b). En años recientes Oithona se ha utilizado como alimento vivo en acuacultura (Santhanam \& Perumal 2012, Vasudevan 2013). Como en la mayoría de copépodos (Tsui 2007), se han registrado migraciones verticales nictimerales de todos los estadios de este género en el estrato superficial (Zamora et al. 2014a, 2014b).

Dentro de los innumerables estudios sobre la comunidad de Copepoda en el Pacífico Americano, pocos consideran a Oithona y menos aún se centran en este género. Los más frecuentes han comprendido la región de California (Estados Unidos y México) durante eventos El Niño y La Niña (Jiménez \& Lara 1988, Hopcroft et al. 2002, Hernández et al. 2005, López \& Palomares 2006, López 2008, Lavaniegos et al. 2012, Dagg et al. 2014). También existen publicaciones de Chile (González et al. 2000, Hidalgo \& Escribano 2001) y Costa Rica (Morales \& Vargas 1995, Morales 1996, 2001).

Aunque se han divulgado algunos resultados de la investigación sobre copépodos en el Pacífico colombiano (López 2012, Jaimes \& López 2014), es la primera vez que se profundiza en el género Oithona, a fin de efectuar un aporte al conocimiento taxonómico y la dinámica ecológica de estos organismos pobremente estudiados en esta área del Pacífico Tropical Oriental (América Central, Galápagos y norte del Perú).

\section{MATERIALES Y MÉTODOS}

\section{Área de estudio}

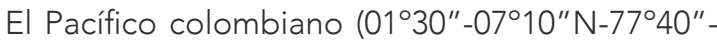
$\left.82^{\circ} 00^{\prime \prime} \mathrm{O}\right)$ hace parte de la Cuenca de Panamá, localizada en aguas de Panamá, Colombia y Ecuador (Fig. 1). Las condiciones hidrodinámicas y termohalinas están definidas por la radiación solar, los patrones eólicos y pluviométricos, las descargas de los ríos y las variaciones de las Corrientes, e.g., Corriente de Humboldt, Contracorriente Ecuatorial, Corriente del Golfo de Panamá y Corriente de Colombia. Estas condiciones son afectadas por el desplazamiento de la Zona de Convergencia Inter-tropical y los eventos cálido El Niño y frío La Niña. En el Pacífico colombiano también se han obervado amplias áreas de surgencia a lo largo del año. En especial alrededor de agosto-septiembre el estrato superior de la columna de agua es más caliente y más profundo que en los primeros seis meses del año (Villegas \& Málikov 2006, Corredor et al. 2011).

\section{Muestreo}

Las muestras se tomaron entre el 23 de junio y el 21 de julio, y entre el 27 de Agosto y el 15 de septiembre de 2001 (segundo periodo lluvioso), en 33 estaciones oceanográficas de las 113 del Estudio Regional del Fenómeno El Niño (ERFEN-Colombia) (Fig. 1). La numeración original de las estaciones se mantuvo para la comparación con otros estudios.

\section{Pacífico colombiano}

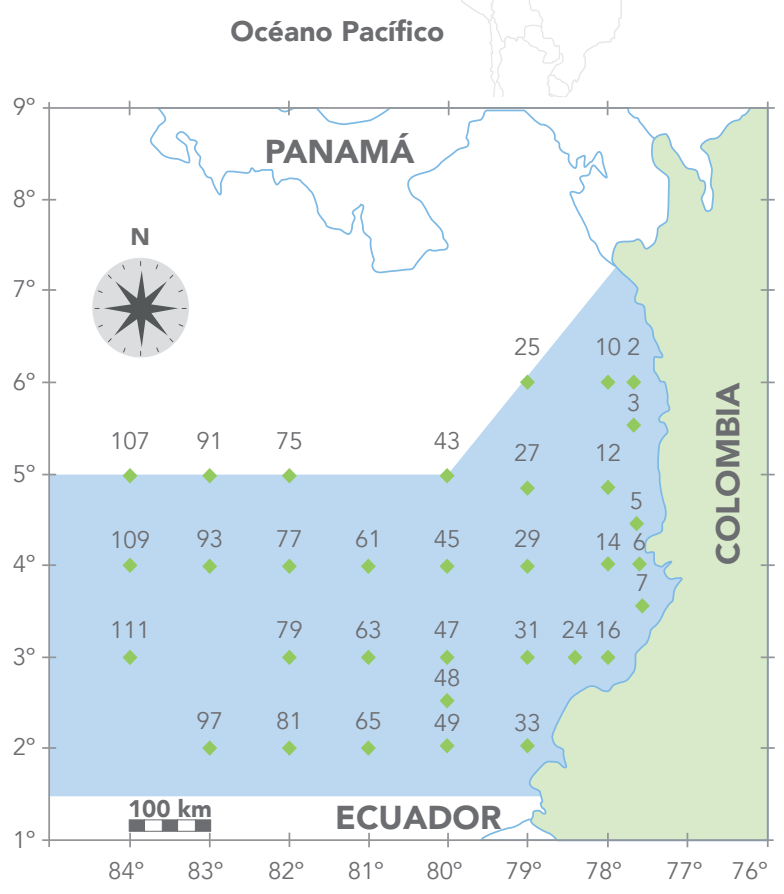

Figura 1. Estaciones oceanográficas del ERFEN-Colombia para el muestreo del mesozooplancton en el Pacífico Colombiano durante el segundo periodo lluvioso de 2001. 
El ERFEN-Colombia sólo contempla una muestra de zooplancton por estación oceanográfica, obtenidas durante el día y la noche, según el derrotero del buque oceanográfico. Los arrastres fueron horizontales de 10 minutos a $1.5 \mathrm{~m} / \mathrm{s}$ en el primer metro superficial y se efectuaron con una red cónica $\varnothing 50 \mathrm{~cm}$, longitud $185 \mathrm{~cm}$, poro de la malla $363 \mu \mathrm{m}$ ), a cuyo aro de entrada se fijó un flujómetro General Oceanics para medir el volumen de agua filtrada. Las muestras se fijaron con formaldehído al 10\% (Suthers \& Rissik 2009).

La temperatura y la salinidad se calcularon mediante los datos obtenidos con un perfilador CTD Sea Bird Electronics-19 en cada estación oceanográfica. Con botellas Niskin de 5 litros se tomaron muestras de agua para efectuar en el laboratorio los análisis de oxígeno disuelto y clorofila-a.

\section{Laboratorio}

Las muestras de mesozooplancton se fraccionaron mediante un divisor Folson, 50\% para obtener los organismos y $50 \%$ para análisis posteriors, ambas submuetras se preservaron con formaldehído al 10\% con borato de sodio (Suthers \& Rissik 2009). En la separación y conteo de los copépodos se usó una cámara Bogorov y un estereomicroscopio. Esta actividad se basó en alícuotas de $10 \mathrm{ml}$ hasta que ca. 200 individuos de las especies predominantes permancieron en ese volumen

Con un microscopio se observaron los principales caracteres diagnósticos contemplados para la identificación de los especímenes, disectados y montados en portaobjetos con glicerina (Reid 2000). Se tuvieron en cuenta: la morfología general (forma del rostrum, prosoma, cefalosoma y urosoma), el desarrollo de la mandíbula y maxilas ylos patrones de distribución, tipo y número de las setas y espinas de los apéndices (Ferrari \& Bowman 1980, Razouls et al. 2014).

Las concentraciones de oxígeno disuelto $(\mathrm{ml} / \mathrm{l})$ se establecieron mediante el método de Winkler y las de clorofila-a $\left(\mathrm{mg} / \mathrm{m}^{3}\right)$ por extracción con acetona (90\%), en ambos casos por espectrofotometría (Clesceri et al. 2001).

\section{Análisis de los datos}

Las abundancias originales se llevaron a número de copépodos $/ \mathrm{m}^{3}$ utilizando la ecuación: $\mathrm{N}=1 \mathrm{~m}^{3}$ * $n / \pi$ * $r^{2}$ * $d$, donde $N=$ abundancia estandarizada, $\mathrm{n}=$ individuos/muestra, $\pi * r^{2}$ d $\mathrm{d}=$ volumen filtrado/ arrastre; $r=$ radio de la red, $d=$ distancia del arrastre (m) (Suthers \& Rissik 2009). La matriz de los valores de abundancia se tranformó con el empleo de la función $\log (x+1)$, siendo $x=$ copépodos $/ \mathrm{m}^{3}$, antes de los análisis para disminuir la magnitud de las diferencias de abundancia entre especies.

Se aplicó un análisis de reducción dimensional, mediante el Análisis de Componentes Principales (ACP) basado en la matriz de correlación, para evaluar las posibles asociaciones entre los seis taxa identificados con la temperatura $\left({ }^{\circ} \mathrm{C}\right)$, la salinidad (ups), el oxígeno disuelto (mg/l), la clorofila-a (mg/ $\mathrm{m}^{3}$ ) y las variaciones según la fase lunar y la hora de cada arrastre, asumiendo las capturas nocturnas entre las 18:01 y las 05:59 h, y las diurnas entre las 06:00 y las 18:00 h. En los análisis estadísticos se empleó el software Statgraphics Plus 5.0.

\section{RESULTADOS Y DISCUSIÓN}

\section{Variables físico-químicas}

Los intervalos generales de temperatura (26.0$\left.29.7^{\circ} \mathrm{C}\right)$, salinidad (27.1-33.6 ups), oxígeno disuelto (3.8-5.4 $\mathrm{ml} / \mathrm{l})$ y clorofila-a $\left(0-3.2 \mathrm{mg} / \mathrm{m}^{3}\right)$ superficiales fueron de condiciones normales, aun cuando en el área el episodio La Niña 1998-2001 se detectó el ultimo año, pero sólo en las capas subsuperficiales (Cadena et al. 2006).

Los promedios/mes señalaron una reducción leve de la salinidad y el oxígeno disuelto, en contraste con el aumento de la salinidad y la clorofila-a, 

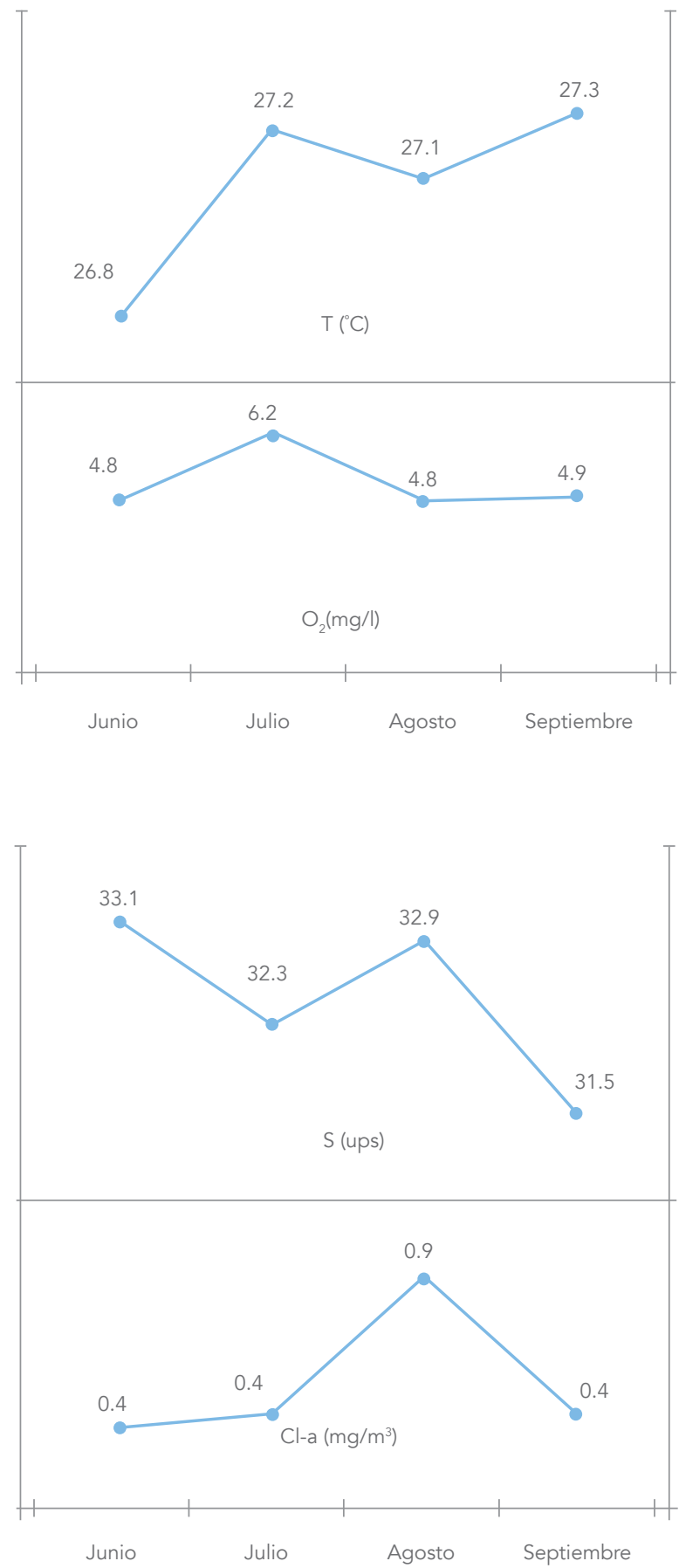

Figura 2. Promedios mensuales de temperatura $\left({ }^{\circ} \mathrm{C}\right)$, salinidad (ups), oxígeno disuelto $(\mathrm{mg} / \mathrm{l})$ y clorofila-a $\left(\mathrm{mg} / \mathrm{m}^{3}\right)$ en la superficie del Pacífico Colombiano en el segundo periodo lluvioso de 2001. esta última con un máximo en agosto (Fig. 2), eventualmente atribuible a aguas de surgencia, proceso común en el Pacífico colombiano (Villegas \& Málikov 2006, Uribe 2003).

\section{Identificación}

En total se encontraron cinco especies epipelágicas: O. plumifera Baird, 1843 (61.8\%), O. rigida Giesbrecht, 1896 (1897) (15.0\%), O. atlantica Farran, 1908 (8.7\%), O. nana Giesbrecht, 1892 (5.9\%) y O. robusta Giesbrecht, 1892 (2.0\%); el 6.7\% restante correspondió a varias especies sin identificar, i. e., Oithona spp.

Pese al número bajo de taxa identificados, se considera un aporte importante, pues la identificación específica sigue siendo un reto debido al tamaño pequeño de estos copépodos, los rasgos morfológicos de diagnóstico sutiles y la descripción de las formas geográficas o variedades. Se recurre con mayor frecuencia a los análisis moleculares, asimismo útiles para caracterizar con precisión las distribuciones biogeográficas de las especies y examinar las relaciones filogenéticas entre ellas (Cepeda et al. 2012). Por supuesto, tales análisis no diferencian las estructuras morfológicas externas básicas para la taxonomía alfa (identificación de especies).

En el Pacífico Americano habitan 18 de las 49 especies de Oithona registradas en el mundo. Por su distribución y hábitat podrían aparecer en el Pacífico colombiano las otras especies no identificadas en este trabajo, salvo O. alvarezi (endémica de la laguna Coyuca, México) (Razouls et al. 2014).

\section{Abundancia de las especies de Oithona}

En primer lugar, es necesario advertir que en la captura de copépodos, sobre todo los más pequeños, el bajo número de especies está influenciado por el poro de las mallas (Morales \& Vargas 1995, Morales 2001, Brugnoli et al. 2004). Por esta razón, la familia Oithonidae e incluso Oithona, a menudo muy 
Tabla 1. Longitud corporal $(\mathrm{mm})$ de las especies de Oithona presentes en el Pacífico Colombiano en el segundo periodo lluvioso de 2001.

\begin{tabular}{|c|c|c|c|}
\hline Especie & Hembras & Machos & Fuente \\
\hline O. atlantica & $\begin{array}{c}\text { 1. } 14-1.43 \\
>1\end{array}$ & & $\begin{array}{l}\text { Nishida (1985) } \\
\text { Takahashi \& Uchiyama (2007) }\end{array}$ \\
\hline O. nana & $\begin{array}{l}0.58-0.72 \\
0.48-0.83 \\
<1 \\
0.57-0.65 \\
0.50-0.80 \\
0.33-0.60\end{array}$ & $\begin{array}{l}0.56-0.66 \\
0.48-0.60\end{array}$ & $\begin{array}{l}\text { Ferrari \& Bowman (1980) } \\
\text { Nishida (1985) } \\
\text { Takahashi \& Uchiyama (2007) } \\
\text { Temperoni (2008) } \\
\text { Vives \& Shmeleva (2010) } \\
\text { Razouls et al. (2014) }\end{array}$ \\
\hline O. plumifera & $\begin{array}{c}1.06-1.28 \\
1.11-1.51 \\
>1 \\
1.13\end{array}$ & $0.59-0.68$ & $\begin{array}{l}\text { Ferrari \& Bowman (1980) } \\
\text { Nishida (1985) } \\
\text { Takahashi \& Uchiyama (2007) } \\
\text { Razouls et al. (2014) }\end{array}$ \\
\hline O. rigida & $\begin{array}{l}0.78-0.83 \\
>1 \\
0.75-0.84\end{array}$ & & $\begin{array}{l}\text { Nishida (1985) } \\
\text { Takahashi \& Uchiyama (2007) } \\
\text { Razouls et al. (2014) }\end{array}$ \\
\hline O. robusta & 1,65 & & Vives \& Shmeleva (2010) \\
\hline
\end{tabular}

abundantes (e. g., Gallienne \& Robins 2001, Paffenhöfer \& Mazzocchi 2003, Hwang et al. 2007, Dagg et al. 2014), han sido históricamente subestimados; no obstante, se ha demostrado que, cuando se utilizan mallas más finas, su abundancia (y a veces su biomasa), pueden ser muy superiores a las de copépodos más grandes (e. g., Turner 2004, Calbet \& Saiz 2005, Makabe et al. 2012). La información sobre las tallas se ha centrado en las hembras y es escasa (Tabla 1), o aún inexistente, como en $\bigcirc$. alvarezi y $\bigcirc$. pacifica.

En el Pacífico colombiano, arratres oblícuos en los primeros $160 \mathrm{~m}$ de profundidad en septiembre de 2007 indicaron el predominio de las tallas de 1.00 a $2.18 \mathrm{~mm}$ en la comunidad de copépodos, sin particularizar por género o especie. Estos resultados corresponden a la malla de $294 \mu \mathrm{m}$ de la red bongo empleada (Jaimes \& López 2014).

Los aspectos mencionados sugieren, en parte, una subestimación del número de especies de Oithona capturadas en el Pacífico colombiano debido a su longitud corporal y a la malla usada (363 $\mu \mathrm{m})$. A lo mismo atribuyeron Morales \& Vargas (1995), Morales (2001) y Brugnoli et al. (2004) la ausencia de especies de este género y de Oncaea en Golfo de Nicoya, Costa Rica. 
Por las diferencias en las mallas y metodologías, sin obviar la frecuencia de los muestreos y las diferentes épocas evaluadas, bastan algunos de los múltiples ejemplos a nivel mundial, para comprender lo inoficioso de las comparaciones objetivas sobre las abundancias de Oithona con trabajos de otras áreas. Sin embargo, ayudan a discernir las tendencias generales con prudencia, pues como bien advierten Hopcroft et al. (2002), las expectativas regionales relacionadas con la perturbación climática no pueden extrapolarse a todas las escalas locales.

El único estudio semejante al presente es el de Tutasi et al. (2011), realizado de la costa de Ecuador a las islas Galápagos durante La Niña 2011, cuando la distribución y abundancia de copépodos mostraron diferencias geográficas claras. Del total de 97 especies, las únicas de Oithona capturadas en esa región (O. plumifera, $O$. spinirostris y $O$. nana) tuvieron abundancias promedio muy bajas $(0.06,0.01 \mathrm{y}$ $0.001 \mathrm{Ind}$./m3, respectivamente).

En el área de estudio en 2001 los valores más altos de Oithona se registraron en junio con tendencia a disminuir cronológicamente, al punto que $O$. nana desapareció en agosto, único mes en que se capturó $O$. atlantica. La única especie permanente fue $O$. plumifera, mientras que $O$. rigida fue exclusiva

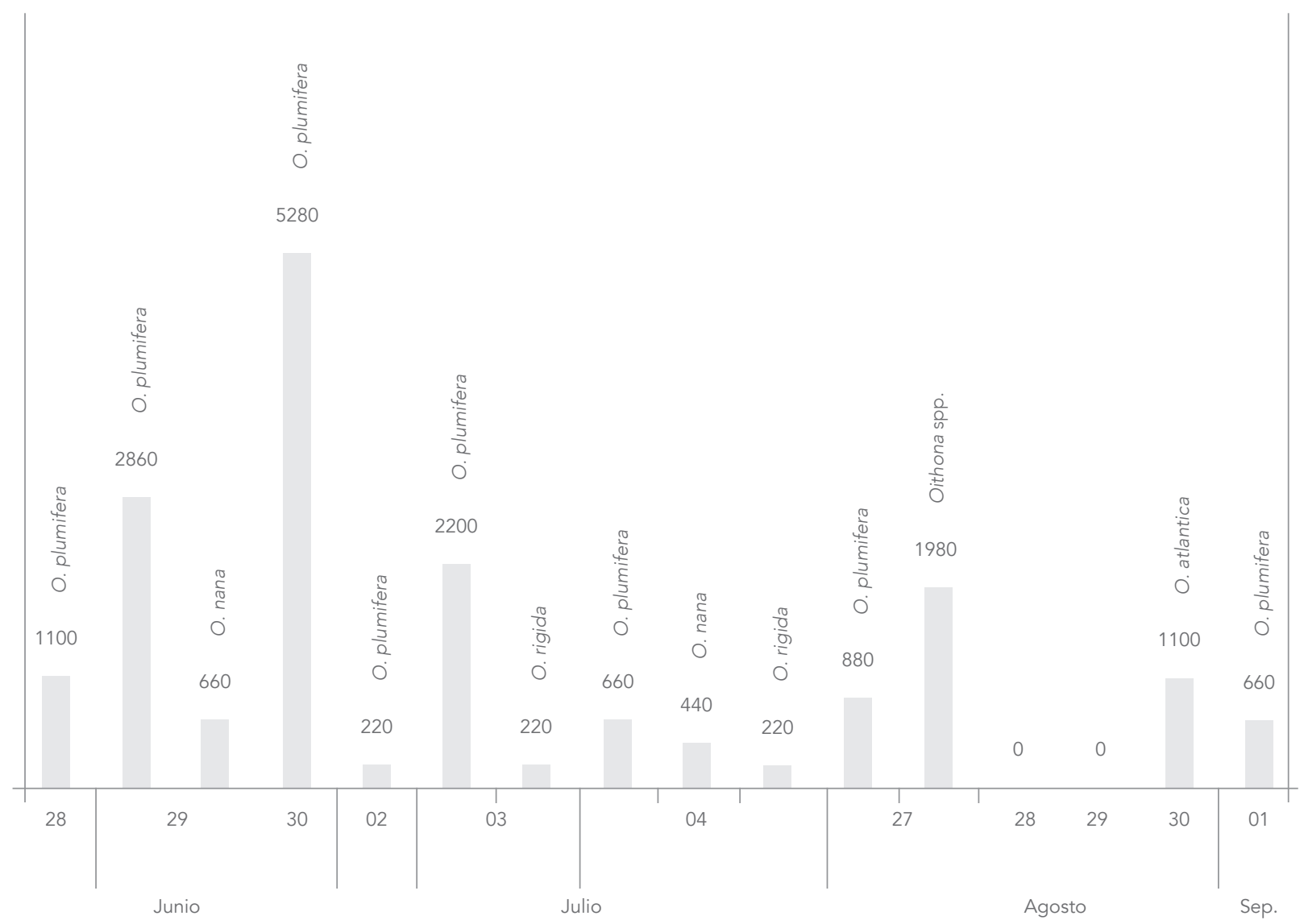

Figura 3. Variación cronológica de la abundancia de las especies de Oithona ( $\left(\mathrm{nd} . / \mathrm{m}^{3}\right)$ halladas en el Pacífico Colombiano en el segundo periodo lluvioso de 2001. 
de julio (Fig. 3). Jaimes \& López (2014) estimaron un promedio general de copépodos de $149 \mathrm{Ind} . / \mathrm{m}^{3}$ en el estrato 0-160 m (arrastres oblícuos).

El predominio de O. plumifera (Tabla 2) no es extraño pues es una de las especies más ubicuas en los océanos del mundo por su gran éxito ecológico, aún en sistemas oligotróficos (Paffenhöfer \& Mazzocchi 2002). O. rigida le secundó en abundancia con gran amplitud y mayor presencia en aguas neríticas. La aparición de O. robusta sólo apareció en aguas oceánicas, mientras que la frecuencia de los demás taxa fue baja.
La falta de información ecológica sobre estas especies en el Pacífico colombiano y áreas vecinas restringe el análisis de estos resultados. Puede mencionarse que el grupo de especies de Oithona identificados en la época de estudio sólo representó $2.1 \%$ del total de la comunidad de copépodos, mientras que el género, y en particular O. plumifera, predominan en otras regiones del Pacífico Americano, como en México. En el canal San Lorenzo (Golfo de California Bahía La Paz) estos taxa son comunes todo el año (Jiménez \& Lara 1988). Allí O. plumifera

Tabla 2. Abundancia de las especies de Oithona ( $\left(n d . / \mathrm{m}^{3}\right)$ en el Pacífico Colombiano en el segundo periodo lluvioso de 2001.

\begin{tabular}{|c|c|c|c|c|c|c|c|c|c|c|c|}
\hline \multicolumn{6}{|c|}{ Zona Nerítica } & \multicolumn{6}{|c|}{ Zona Oceánica } \\
\hline 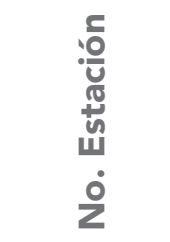 & $\frac{0}{\frac{0}{0}}$ & $\frac{\frac{\pi}{0}}{\frac{\pi}{0}}$ & 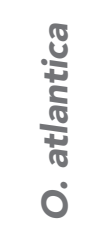 & 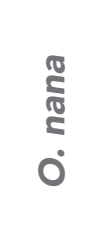 & $\begin{array}{l}\frac{\dot{0}}{\alpha} \\
\frac{1}{0} \\
\frac{0}{5} \\
\frac{0}{1} \\
\dot{0}\end{array}$ & 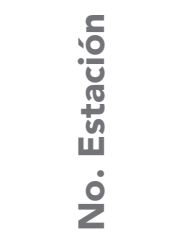 & $\frac{\frac{0}{0}}{\frac{5}{5}}$ & $\frac{\pi}{\frac{\pi}{0}}$ & 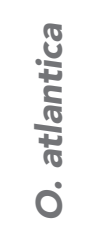 & $\begin{array}{c}\underset{0}{S} \\
\underset{\mathbb{C}}{\leq} \\
0\end{array}$ & $\begin{array}{l}\mathbb{d} \\
\underline{n} \\
0 \\
0 \\
0 \\
0 \\
0\end{array}$ \\
\hline 2 & & 880 & & & & 25 & & & & & \\
\hline 3 & & 2860 & & & & 27 & 1540 & & 660 & & \\
\hline 5 & & & & & & 29 & 1430 & 220 & 440 & & \\
\hline 6 & & 1760 & & & & 31 & 1320 & & & & \\
\hline 7 & & & & & & 43 & & & & & \\
\hline 10 & & & & & & 45 & 660 & 220 & & & \\
\hline 12 & & 2420 & & & 660 & 47 & & & & & \\
\hline 14 & 2420 & & 2200 & & & 48 & & & & & \\
\hline 16 & 1100 & & & 660 & & 49 & 1540 & & & & \\
\hline 24 & 660 & & & 220 & & 61 & 1320 & 880 & & & \\
\hline 33 & 1100 & & & 440 & 1540 & 63 & 220 & & & & \\
\hline & & & & & & 65 & 660 & & & & \\
\hline & & & & & & 75 & 660 & & & & \\
\hline & & & & & & 77 & 440 & & & & \\
\hline & & & & & & 79 & 880 & & & & \\
\hline & & & & & & 81 & 1980 & & & & \\
\hline & & & & & & 91 & 2200 & & & & \\
\hline & & & & & & 93 & 1100 & & & & \\
\hline & & & & & & 97 & 2420 & & & 880 & 1100 \\
\hline & & & & & & 107 & 2640 & & & & \\
\hline & & & & & & 109 & 1540 & & & 660 & \\
\hline & & & & & & 111 & 1320 & 1100 & & & \\
\hline Total & 5280 & 7920 & 2200 & 1320 & 2200 & Total & 23870 & 2420 & 1100 & 1540 & 1100 \\
\hline Promedio & 1320 & 1980 & 2200 & 440 & 1100 & Promedio & 1326 & 605 & 550 & 770 & 1100 \\
\hline DE & 762 & 861 & & 220 & 622 & $\mathrm{DE}$ & 680 & 454 & 156 & 156 & \\
\hline
\end{tabular}


está influida en gran extensión por la Corriente del Golfo de California y también es frecuente en la Bahía Los Ángeles, junto con O. nana (Lavaniegos et al. 2012), la cual predomina en época lluviosa, similar a lo observado en el Pacífico colombiano, aunque no se consideró ese tipo de ambientes.

Se han observado máximos de abundancia en el verano, en otras regiones, donde el género Oithona es común, e. g., aguas polares argentinas (Zamora 2014a), similar a lo observado en el Pacífico colombiano en los meses evaluados. O. rigida es la especie más común en las aguas costeras de Parangipettai (India), hecho explicado por su alta capacidad reproductiva, desoves continuos y crecimiento rápido (Vasudevan 2013).

En todo el mundo, incluido el Pacífico ecuatorial oriental $\left(22^{\circ} \mathrm{S}-8^{\circ} \mathrm{N}\right.$ centro del Pacífico sur al Golfo de Panamá), la comunidad zooplanctónica experimenta cambios espaciales debido a la variabilidad ambiental estacional. El número total de copépodos cambia según la surgencia ecuatorial y la advección horizontal del agua de surgencia frente al Perú, que al intensificarse desencadenan las abundancias máximas de julio a septiembre. Sobre todo durante los episodios El Niño se han observado cambios de índole variada en la comunidad de copéopodos en el Golfo de Panamá (Dessier \& Donguy 1987).

Al igual que esa extensa región, el Pacífico colombiano muestra una gran heterogeneidad oceanográfica y ecológica que repercute sobre el fitoplancton (Uribe 2003) y los copépodos (López 2012, Jaimes \& López 2014). Tal heterogeneidad está modulada por el desplazamiento de la Zona de Convergencia Intertropical, las corrientes, las amplias zonas de surgencia y periódicamente El Niño y La Niña (Villegas \& Málikov 2006, Corredor et al. 2011). Pese a este esbozo oceanográfico, porque tampoco se ha profundizado en estos aspectos, ni se tomaron datos sobre las corrientes, y ante la falta de datos sobre Oithona anteriores al periodo de estudio en el Pacífico colombiano, no pudo establecerse si los taxa identificados son residentes o frecuentes, ni si la composición y abundancia cambia durante el año.

\section{Abundancia día: noche \& fases lunares}

En general la abundancia relativa fue un poco mayor en la noche que en el día (56.3 vs. $43.7 \%$ ). 0. robusta y $O$. atlantica sólo se capturaron en horas nocturas, en las que predominó $O$. rigida; las demás especies fueron más abundantes en el día. O. plumifera y $O$. nana, exhibieron abundancias similares durante luna llena y cuarto creciente, mientras que O.rigida y $O$. atlantica fueron más distintivas en la primera fase lunar y $O$. robusta sólo apareción en luna nueva. Las demás especies del género no identificadas prdominaron en cuarto creciente (Fig. 3).

Los cambios nictimerales de la abundancia de copépodos se han explicado, entre otros factores, por las migraciones verticales en la columna de agua, para evitar la presión trófica y búsqueda de alimento, de forma que en la noche pueden aumentar considerablemente en la superficie marina (Tsui 2007).

En el caso de Oithona, las referencias sobre este tipo de desplazamientos son pocas y disímiles, e. g., González et al. (2000) refieren que los ciclopoideos pequeños (Corycaeus, Oncaea y Oithona) se mantienen en los primeros $50 \mathrm{~m}$ de la columna de agua. Hwang et al. (2010) señalan que las diferencias día (06:00-18:00 h): noche (18:00-06:00 h) son perceptibles sólo en la superficie, donde $O$. rigida y Oithona spp., aparecieron sólo en la noche. Esto muestra alguna coincidencia con los registros en el Pacífico colombiano, por tanto allí estas especies se capturaron en mayor medida en horas de oscuridad. En cambio, Zamora et al. (2014a, 2014b) observaron una distribución batimétrica amplia de Oithona, en especial 0-100 m y hasta $200 \mathrm{~m}$, aunque los nauplios exhiben una distribución más somera; además que las variaciones cambian según la estación, con una distribución más profunda de las hembras adultas en invierno. 


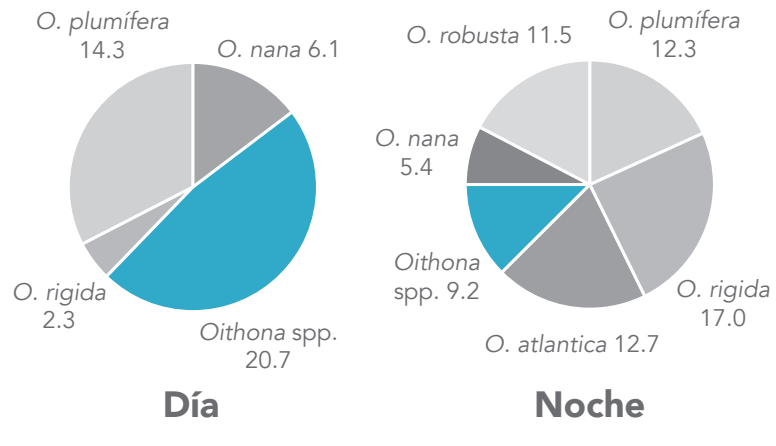

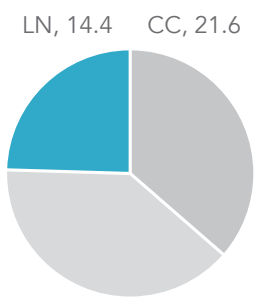

LL, 22.6

\section{O. plumífera}

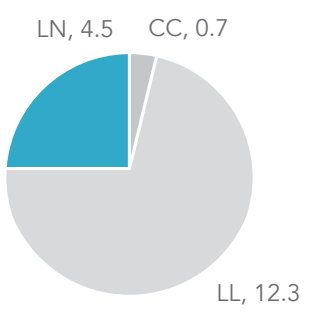

\section{O. rigida}

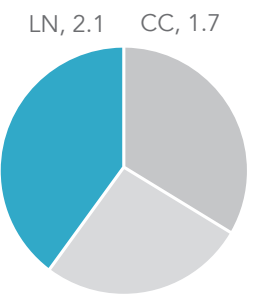

LN, 1.4

O. nana

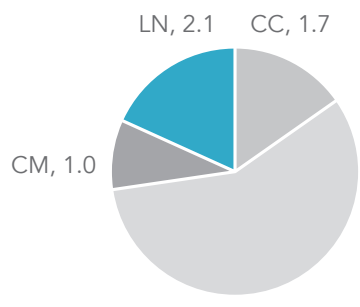

$\mathrm{LL}, 6.5$

O. atlantica

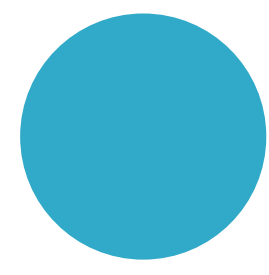

LN, 1.7

O. robusta

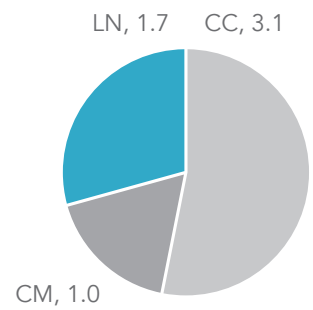

Oithona spp.
Figura 3. Abundancia relativa (\%) nictimeral y por fase lunar de las especies de Oithona halladas en el Pacífico Colombiano en el segundo periodo lluvioso de 2001. $C C=$ Cuarto Creciente, $C M=$ Cuarto Menguante, LN=Luna Nueva, LL= Luna Llena.
Basados en datos históricos de abundancia entre 0-200 m en aguas subtropicales, Hernández et al. (2001) establecieron que el estrato 0-100 m es el más afectado por los cambios en la iluminación lunar. Además, que durante luna llena en la superficie aumentan los copépodos y otras formas epipelágicas, favorecidos por la disminución del macrozooplancton y el micronecton, que no alcanzan esa capa con el fin de evitar la depredación beneficiada por el relativamente alto nivel de iluminación; durante la luna nueva suele ocurrir lo contrario.

En el Pacífico colombiano se observó algo similar a lo anterior en el caso de O. plumifera, O. rigida y $O$. atlantica, pero no se puede imputar sólo a la depredación, pues no se realizaron estudios tróficos. Las diferencias circadianas también ha sido observadas en la comunidad general de copépodos, capturados en la superficie del Pacífico colombiano, con mayor intensidad en cuarto menguante, aunque con variaciones interanuales, atribuibles a la composición taxonómica y ontogénica (López 2012). Arrastres oblicuos en los primeros $160 \mathrm{~m}$ de profundidad han indicado un aumento durante luna llena, sin evidenciar migraciones verticales, por el tipo de muestro (oblicuo, no estratificado) (Jaimes \& López 2014).

\section{Análisis estadísticos}

En el ACP los primeros tres componentes explicaron apenas el $44.01 \%$ de la varianza, comparado con un análisis de factores en aguas de Ecuador en septiembre-octubre de 2001, el cual reveló que 91\% de la variabilidad de los datos en la distribución y abundancia de las especies de copépodos fue explicada por la temperatura y la salinidad. No obstante, el bajo número de estaciones evaluadas (41) no permitió determinar cuál de esas variables causó un mayor sobre estos organismos (Tutasi et al. 2011). En la presente evaluación también se debe tener cautela, por el número limitado de estaciones oceanográficas (33). La aplicación de un análisis de factores fue menos útil que el ACP. 
El primer componente del ACP se explicó con el mayor éxito poblacional de O. plumifera y $O$. rigida en términos de su abundancia (61.8 y 15.0\% respectivamente), la temperatura, el $\mathrm{O}_{2}$ y la salinidad (Fig. 4), análogo a los resultados de Tutasi et al. (2011) en aguas ecuatorianas alrededor de la misma época. En el componente 2 se corroboró la influencia térmica sobre $O$. plumifera y se destacó la relevancia de la clorofila-a, la hora de captura y la temperatura, en relación con O. robusta. En C3 la influencia lunar mostró la mayor asociación positiva, seguida de la clorofila-a y O. nana (inversa), reconocida consumidora de fitoplancton (Lampitt \& Gamble 1982), lo cual sugiere la importancia de estas variables en el área de estudio.

Por extensión, las asociaciones inversas entre Oithona y la clorofila-a acaso se relacionen con el mayor pastoreo que ejerce frente a otros copépodos más grandes, sobre todo cuando son abundantes (Paffenhöfer 1993).

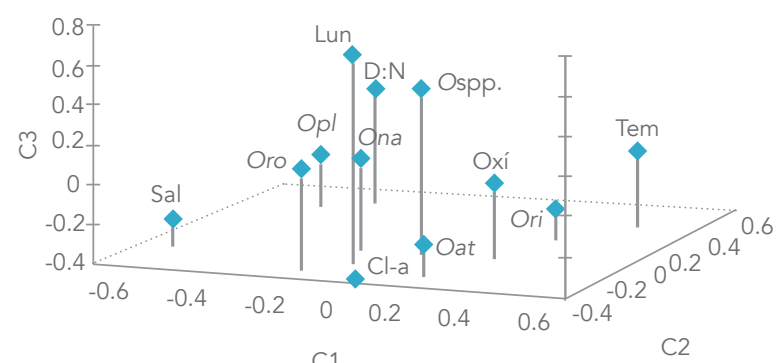

Figura 4. Asociación de variables (especies) y observaciones (estaciones) de los tres primeros componentes $(\mathrm{C} 1, \mathrm{C} 2, \mathrm{C} 3)$ resultantes del ACP en el Pacífico Colombiano. Oat= O. atlantica, Ona= O. nana, $O p l=O$. plumifera, Ori= O. rigida, Oro= O. robusta, Ospp.= Oithona spp., Tem $=$ temperatura, Sal= salinidad, $\mathrm{Cl}-a=$ clorofila- $a$, Lun= fases lunares, $\mathrm{D}: \mathrm{N}=$ variación día: noche.

Las correlaciones poco signigificativas en el ACP entre la abundancias y la clorofila-a indicarían que recursos alimentarios diferentes del fitoplancton contribuirían a la dieta de Oithona spp., en el Pacífico colombiano, similar a lo observado por
Zamora et al. (2014a, 2014b), así como para otros ciclopoideos pequeños, como Oncaea venusta y O. media (Turner 2004).

Considerando que estos organismos han sido poco estudiados en el Pacífico colombiano, esta es una contribución importante a su conocimiento. La mayor relevancia de este estudio es que por primera vez que se profundiza en algunos atributos ecológicos de Oithona en Colombia.

Sin embargo, los resultados presentados deben considerarse introductorios para ayudar a comprender la dinámica ecológica de las especies de Oithona en el Pacífico colombiano, por varios motivos: 1. La selectividad de la red empleada, pues seguramente afectó la composición de especies y la estimación de su abundancia. En el futuro deben emplearse mallas más finas para estudios de Cyclopoida. 2. El bajo número de estaciones. 3. Debido a las diferentes afinidades biogeográficas de las especies de copépodos que se ajustan a las sucesiones estacionales (López \& Palomares 2006), es muy probable que haya diferencias intra- e interanuales, lo cual requiere estudios con muestreos a lo largo del año, aunque en el marco del ERFEN-Colombia esto no ha sido por los costos elevados. 4. Definitivamente, el principal problema en la interpretación de los datos se relaciona con la alta variabilidad espacial y cronológica del zooplancton y, por supuesto, de los copépodos, debida a la heterogeneidad del medio marino y los cambios oceanográficos contínuos (Hernández et al. 2001).

En trabajos futuros se sugiere: 1. Establecer las diferencias de tamaño de $q$ y $\hat{\jmath}$, debido al dimorfismo sexual en Oithona (aunque no pronunciado y evidente sólo en algunas especies) (Razouls et al. 2014), pues por lo general se captura un mayor número de hembras (Ferrari \& Bowman 1980), lo cual quizás ocurrió en el Pacífico colombiano, pero no pudo establecerse, pues los especímenes no 
se cuantificaron por sexo. 2. Evaluar el papel de Oithona en retardar el flujo vertical del material fecal del zooplankton, relevante en otras áreas (González \& Smetacek 1994, Dagg et al. 2014). 3. Dada la importancia de las fases larvarias para el éxito del reclutamiento de las poblaciones, es necesario entender la variabilidad e influencia ambiental (temperatura, oferta alimentaria, etc.) y los factores inherentes (edad, tamaño, etc.) sobre la actividad metabólica de Oithona, para comprender su capacidad para explotar los diferentes ecosistemas marinos (Almeda et al. 2011). 4. Considerar su ecología alimentaria, su papel como presa en los niveles tróficos superiores y las particularidades de su biología reproductiva, lo que podría influir en su comportamieto trófico (Turner 2004).

Estos aspectos son poco conocidos en el resto del mundo y el Pacífico colombiano, en especial el espectro trófico del género y más aún de las especies. Esta información contribuiría a orientar las prospecciones futuras de zooplancton, al considerar elementos del nano- y picoplancton no estudiados aún en esa área. Su carencia dificulta explicar las abundancias y su distribución, dependientes del alimento disponible en el medio, además de las condiciones abióticas. Por ende, se compendia lo siguiente:

El género Oithona puede definirse como omnívoro, por su espectro alimentario amplio, aunque estudios recientes cuestionan su papel en el consumo de heces de zooplancton (=pellets fecales) (Reigstad et al. 2005, Iversen \& Poulsen 2007), lo cual tiene un papel relevante al retardar el flujo vertical de ese material (González \& Smetacek 1994, Dagg et al. 2014).

De las especies capturadas en el Pacífico colombiano, se sabe que $O$. nana tiene una dieta muy variada que abarca el fitoplancton y una tasa metabólica baja, aspectos que son considerados estrategias adaptativas para mantener sus niveles poblacionales todo el año (Lampitt \& Gamble 1982).

En otras regiones, donde las especies de Oithona son habituales (como la zona polar de Argentina), se han observado máximos asociados al aumento de la biomasa de protozooplancton y la temperatura, i. e., en verano, enfatizando la importancia clave de estos copépodos pequeños, especialmente cuando los más grandes no están presentes en el estrato superficial (Zamora 2014a).

\section{AGRADECIMIENTOS}

Este manuscrito es uno de los productos del proyecto CIAS-1181: Copepoda: herramienta para evaluar la dinámica del mesozooplancton en el Océano Pacífico Colombiano, financiado por la Vicerrectoría de Investigaciones de la Universidad Militar Nueva Granada. La participación en la fase de campo fue posible gracias a la Dirección General Marítima (DIMAR) y su Centro de Investigaciones Oceanográficas e Hidrográficas del Pacífico, que además suministraron los datos brutos de temperatura, salinidad, oxígeno disuelto y clorofila-a. 


\section{REFERENCIAS}

1. Almeda R, Alcaraz M, Calbet A \& Saiz E. 2011. Metabolic rates and carbon budget of early developmental stages of the marine cyclopoid copepod Oithona davisae. Limnology and Oceanography, 56: 403-414.

2. Brugnoli E., Díaz E., Delfino M., Morales Á. \& Dominici A. 2004. Composition of the zooplankton community, with emphasis in copepods, in Punta Morales, Golfo de Nicoya, Costa Rica. Revista de Biología Tropical, 52(4): 897-902.

3. Cadena M, Ortiz J, Málikov I, Reyna J, Pabón D \& Devis A. 2006. Relationship between the 1997/98 El Niño and 1999/2001 La Niña events and oil palm tree production in Tumaco, Southwestern Colombia. Advances in Geosciences, 6(1): 195-199.

4. Calbet A \& Saiz E. 2005. The ciliate-copepod link in marine ecosystems. Aquatic Microbial Ecology, 38: 157-167.

5. Cepeda GD, Blanco-Bercial L, Bucklin A, Berón CM \& Viñas MD. 2012. Molecular systematic of three species of Oithona (Copepoda, Cyclopoida) from the Atlantic Ocean: Comparative analysis using $28 \mathrm{~S}$ rDNA. PLoS ONE 7(4): e35861. doi:10.1371/journal.pone.0035861. Consulta 21 de diciembre de 2014.

6. Clesceri LS, Greenberg AE \& Eaton AD. 2001. Standard methods for the examination of water and wastewater. American Public Health Association, Washington, $1325 \mathrm{p}$.
7. Corredor A, Acosta A, Gaspar P \& Calmettes B. 2011. Variation in the surface currents in the Panama bight during El Niño and La Niña events from 1993 to 2007. Boletín de Investigaciones Marinas y Costeras, 40 (Supl. Esp.): 33-56.

8. Dagg MJ, Jackson GA \& David M. Checkley Jr. DM. 2014. The distribution and vertical flux of fecal pellets from large zooplankton in Monterey bay and coastal California. Deep Sea Research Part I: Oceanographic Research Papers, 94: 72-86.

9. Dessier A \& Donguy JR. 1987. Response to El Niño signals of the epiplanktonic copepod populations in the eastern tropical Pacific. Journal of Geophysical Research, 921: 14393-14403.

10. Ferrari FD \& Bowman TE. 1980. Pelagic copepods of the family Oithonidae (Cyclopoida) from the east coasts of Central and South America. Smithsonian Contributions to Zoology, 312:27.

11. Ferrari FD \& Orsi J. 1984. Oithona davisae, new species, and Limnoithona sinensis (Burkckhard, 1912) (Copepoda: Oithonidae) from the Sacramento-San Joaquin Estuary, California. Journal of Crustacean Biology 4(1): 106-126.

12. Gallienne CP \& Robins DB. 2001. Is Oithona the most important copepod in the world's oceans? Journal of Plankton Research, 23: 1421-1432.

13. González HE \& Smetacek V. 1994. The possible role of the cyclopoid copepod Oithona in 
retarding vertical flux of zooplankton faecal material. Marine Ecology Progress Series, 113: 233-246.

14. González HE, Sobarzo M, Figueroa D \& Nöthig EM. 2000. Composition, biomass and potential grazing impact of the crustacean and pelagic tunicates in the northern Humboldt Current area off Chile: differences between El Niño and non-El Niño years. Marine Ecology Progress Series, 195: 201-220.

15. Hernández S, Almida L, Arístegui J, Fernández ML \& García J. 2001. Zooplankton abundance in subtropical waters: Is there a lunar cycle? Scientia Marina, 65(Suppl. 1): 59-63.

16. Hernández S, Palomares R, López GA, Esqueda GM \& Pacheco MR. 2005. Riqueza específica de copépodos en Bahía Magdalena, Baja California Sur, México. Anales del Instituto de Biología, Universidad Nacional Autónoma de México, Serie Zoología 75(2): 253-270.

17. Hidalgo P \& Escribano R. 2001. Succession of pelagic copepod species in coastal waters off northern Chile: the influence of the 1997-98 El Niño. Hydrobiologia, 453-454(1): 153-160.

18. Hopcroft RR, Clarke C \& Chávez FP. 2002. Copepod communities in Monterey Bay during the 1997-1999 El Niño and La Niña. Progress in Oceanography, 54: 251-264.

19. Hwang JS, Kumar R, Dahms HU, Tseng LC \& Chen QC. 2007. Mesh size affects abundance estimates of Oithona spp. (Copepoda, Cyclopoida) Crustaceana, 80(7): 827-837.

20. Hwang JS, Kumar R, Dahms HU, Tseng LC \& Chen QC. 2010. Interannual, seasonal, and diurnal variations in vertical and horizontal distribution patterns of 6 Oithona spp. (Copepoda: Cyclopoida) in the South China Sea. Zoological Studies, 49(2): 220-229.

21. Iversen MH \& Poulsen LK. 2007. Coprohexy, coprophagy, and coprochaly in the copepods Calanus helgolandicus, Pseudocalanus elongatus and Oithona similis. Marine Ecology Progress Series, 350: 79-89.

22. Jaimes JC \& López RH. 2014. Biomasa y abundancia de Copepoda (Crustacea) en aguas superficiales del océano Pacífico colombiano durante septiembre de 2007. Revista de Biología Marina y Oceanografía, 49(1): 31-41.

23. Jiménez JC \& Lara JR. 1988. Zooplankton biomass and copepod community structure in the Gulf of California during the 1982-1983 El Niño event. CalCOFI Report, 29: 122-128.

24. Lampitt RS \& Gamble JC. 1982. Diet and respiration of the small planktonic marine copepod Oithona nana. Marine Biology, 66(2): 185-190.

25. Lavaniegos BE, Heckel G \& Ladrón de Guevara P. 2012. Variabilidad estacional de copépodos y cladóceros de Bahía de los Ángeles (Golfo de California) e importancia de Acartia clausi como alimento del tiburón ballena. Ciencias Marinas, 38(1A): 11-30. 
26. Lonsdale DJ, Caron DA, Dennett MR \& Schaffner R. 2000. Predation by Oithona spp. on protozooplankton in the Ross Sea, Antarctica. Deep-Sea Research, 47: 3273-3283.

27. López GA \& Palomares R. 2006. Estructura de la comunidad de copépodos en Bahía Magdalena, México, durante El Niño 1997-1998. Revista de Biología Marina y Oceanografía, 41(1): 63-76.

28. López RH. 2012. Distribución y abundancia de copépodos pelágicos en el Pacífico colombiano. Revista Facultad de Ciencias, 8(1): 108-131.

29. Makabe R, Tanimura A \& Fukuchi M. 2012. Comparison of mesh size effects on mesozooplankton collection efficiency in the Southern Ocean. Journal of Plankton Research, 34(5): 432-436.

30. Morales A \& Vargas JA. 1995. Especies comunes de copépodos (Crustacea: Copepoda) pelágicos del Golfo de Nicoya, Costa Rica. Revista de Biología Tropical, 43: 207-218.

31. Morales A. 1996. Checklist of copepods from Gulf of Nicoya, Coronado Bay and Golfo Dulce, Pacific coast of Costa Rica, with comments on their distribution. Revista de Biología Tropical, 44: 103-113.

32. Morales A. 2001. Biodiversidad marina en Costa Rica, los microcrustáceos: Subclase Copepoda (Crustacea: Maxillopoda). Revista de Biología Tropical, 49: 115-133.

33. Nishida S. 1985. Taxonomy and distribution of the family Oithonidae (Copepoda, Cyclopoida) in the Pacific and Indian oceans. Bulletin of the Ocean Research Institute, 20: 1-167.

34. Paffenhöfer GA \& Mazzocchi MG. 2002. On some aspects of the behaviour of Oithona plumifera (Copepoda: Cyclopoida). Journal of Plankton Research, 24: 129-135.

35. Paffenhöfer GA \& Mazzocchi MG. 2003. Vertical distribution of subtropical epiplanktonic copepods. Journal of Plankton Research, 25: 1139-1156.

36. Paffenhöfer GA. 1993. On the ecology of marine cyclopoid copepods (Crustacea, Copepoda). Journal of Plankton Research, 15: 37-55.

37. Razouls C, de Bovée F, Kouwenberg J \& Desreumaux N. 2014. Diversity and geographic distribution of marine planktonic copepods. http://copepodes.obs-banyuls.fr/en. Consulta 04 de diciembre de 2014.

38. Reigstad M, Wexels C \& Svensen C. 2005. The fate of copepod faecal pellets and the role of Oithona spp. Marine Ecology Progress Series, 304: 265-270.

39. Santhanam P \& Perumal P. 2012. Developmental biology of brackishwater copepod Oithona rigida Giesbrecht: A laboratory investigation. Indian Journal of Geo-Marine Sciences, 42(2): 236-243.

40. Suthers IM \& Rissik D. (Eds.). 2009. Plankton: A guide to their ecology and monitoring for water quality. CSCIRO Publishing, Collingwood, $272 \mathrm{p}$.

41. Takahashi T \& Uchiyama I. 2007. Morphology of the naupliar stages of some Oithona 
species (Copepoda: Cyclopoida) occurring in Toyama Bay, southern Japan Sea. Plankton and Benthos Research, 2(1): 12-27.

42. Temperoni B. 2008. Variación estacional de la producción secundaria de Oithona nana Copepoda Cyclopoidea) en aguas costeras bonaerenses. Tesis Licenciatura en Ciencias Biológicas, U. Nacional de Mar del Plata, Mar del Plata, 73 p. www.oceandocs.org/bitstream/1834/3131/1/Temperoni_2008.pdf. Consulta 06 de enero de 2015.

43. Tsui N. 2007. Vertical migration of marine copepods in the Galapagos Islands in relation to size and color. Report School of Oceanography, University of Washington, Washington, $26 \mathrm{p}$.

44. Turner JT. 1986. Zooplankton feeding ecology: contents of fecal pellets of the cyclopoid copepods Oncaea vetusta, Corycaeus amazonicus, Oithona plumifera, and O. simplex from the Northern Gulf of Mexico. Marine Ecology, 7: 289-302.

45. Turner JT. 2004. The importance of small planktonic copepods and their roles in pelagic marine food webs. Zoological Studies, 43(2): 255-266.

46. Tutasi P, Palma S \& Cáceres M. 2011. Epipelagic copepod distributions in the eastern equatorial Pacific during the weak La Niña event of 2001. Scientia Marina, 75(4): 791-802.
47. Uribe HJ. 2003. Relación entre las condiciones ambientales y la comunidad fitoplanctónica (diatomeas y dinoflagelados) de la cuenca del Pacífico colombiano (1996-2001). Tesis Facultad de Biología Marina, Universidad de Bogotá Jorge Tadeo Lozano, Bogotá, 89 p.

48. Vasudevan S, Arulmoorthy MP, Gnanamoorthy P \& Ashok P. 2013. Intensive cultivation of the calanoid copepod Oithona rigida for mariculture purpose. International Journal of Pharmacy and Biological Sciences, 3(4): 317-323.

49. Villegas N \& Málikov I. 2006. Modelación de la estructura dinámica de las aguas de la cuenca del Pacífico colombiano. Boletín Científico CCCP, 13: 97-114.

50. Vives F \& Shmeleva AA. 2010. Fauna ibérica. Crustacea, copépodos marinos II. Non Calanoida. Vol. 33. Ed. Consejo Superior de Investigaciones Científicas, Madrid, $492 \mathrm{p}$.

51. Zamora S, Kjellerup S, Swalethorp R, Saiz E \& Nielsen TG. 2014a. Population dynamics and production of the small copepod Oithona spp. in a subarctic fjord of West Greenland. Polar Biology, 37(7): 953-965.

52. Zamora S, Mckinnon DA \& Saiz E. 2014b. Feeding and egg production of Oithona spp. in tropical waters of North Queensland, Australia. Journal of Plankton Research, 36 (4): 10471059. doi: 10.1093/plankt/fbu039. Consulta 08 de diciembre de 2014. 